\title{
Digital in-line holography for the characterization of two phase flows in astigmatic systems
}

\author{
Matthias PL SENTIS ${ }^{1}$, Fabrice LAMADIE ${ }^{1 *}$, Fabrice ONOFRI ${ }^{2}$, Laurent BRUEL ${ }^{3}$, Sophie CHARTON ${ }^{1}$ \\ 1 CEA, DEN, DTEC, SGCS, F-30207 Bagnols-sur-Cèze, France \\ 2 Aix-Marseille Université, CNRS, IUSTI, UMR 7343, 13453 Marseille Cedex 13, France \\ 3 CEA, DEN, DTEC, SEPE, F-30207 Bagnols-sur-Cèze, France \\ Author e-mail address: fabrice.lamadie@cea.fr \\ Abstract: This study details the characterization of two phase flows using digital holography in \\ astigmatic systems through a generalized convolution method derived from the Collins formula. \\ OCIS codes: (090.1995) Digital holography, (050.1940) Diffraction, (120.3940) Metrology
}

\section{Introduction}

Digital Holography (DH) is well-known for allowing, in a single shot, the complete 3D reconstruction of any particulate flow. As highlighted by Lamadie et al. [1], DH involves a very simple measurement setup, that is not sensitive to vibrations and to most typical environmental factors, and is therefore particularly suitable for the characterization of two phase flows. The authors demonstrated the feasibility of the measurements for a liquid/liquid flow, considering a cuboid flow cell. However, most standard devices designed for chemical engineering (for instance pulsed columns, centrifugal extractor, etc.) exhibit a cylindrical geometry inducing astigmatism, thus rendering the classical hologram simulation and focusing methods, inappropriate.

Two main approaches are possible to tackle astigmatism. The first one, introduced by Collins et al. in 1970 [2] is a general expression of the Rayleigh-Sommerfeld integral, including optical system in paraxial approximation thanks to an ABCD transfer matrix. It gives the relationship between the output and input of the complex amplitude distributions of the light field passing through an ABCD optical system. In this context, Rayleigh-Sommerfeld diffraction integral could be analytically expressed, as proved by Verrier et al. [3] or used through a generalized Fresnel Transform (GFT) propagator as described by Palma et al. [4]. The second approach, introduced by Ozaktas et al. [5], relies on the Fractional Fourier Transform (FrFT) properties. According to Ozaktas, all quadratic phase systems (QPS) can be interpreted as a magnified FrFT with a phase curvature.

In this contribution, the characterization of a two-phase flow in a cylindrical cell using DH is described. The first section is devoted to hologram simulation in astigmatic optical system through the GFT propagator and a supplementary Generalized Impulse Response (GIR) propagator. The second section introduces a Best Fitting Approach (BFA) based on GFT and GIR propagators to retrieve the 3D position and size of spherical particles. To improve the positioning of the particles along the optical axis $\mathrm{z}$, a focusing method based on the maximal intensity position of the photonic jet is developed.

\section{Hologram simulation in astigmatic geometry}

Astigmatic optical systems exhibit different properties along the two axes ( $x$ and $y$ ) perpendicular to the direction of propagation $z$. In digital holography, focusing methods usually involve a numerical model for the simulation of the holograms. While in free space this point is easily achieved using a Fresnel Transfer (FT) function or an Impulse Response (IR) propagator [6], this approach is no longer applicable to astigmatic optical systems. Indeed, for such system it is mandatory to differentiate the propagation in $x$ and $y$ directions, and accordingly the kernel functions of the propagators must be asymmetrical.

As mentioned in the introduction, in the paraxial approximation all optical systems can be described by an ABCD matrix [7]. To deal with astigmatic optical systems, this approach is particularly relevant as it allows to use two different matrices $A B C D x$ and $A B C D x$ to describe the system the along $x$ and $y$ axis respectively.

In free space, the Rayleigh-Sommerfeld integral describes the diffracted amplitude $A_{z}$ at distance $z$ for a monochromatic wave. In astigmatic optical systems, the diffracted amplitude can be expressed through the Collins formula [2, 4]. This formula is a generalization of the free space diffraction integral for an optical system represented by an $A B C D$ matrix in the paraxial approximation. Following this formula, it reads for the wave amplitude $A_{z}$ :

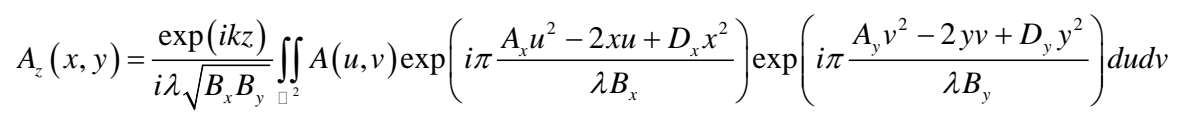


with $A(u, v)$ the complex amplitude distribution of the light field before propagation, $\lambda$ the wavelength, $z$ the distance of propagation along the optical axis and $k=2 \pi / \lambda$ the wave number. The coefficients $A_{x}, B_{x}, C_{x}, D_{x}$ and $A_{y}, B_{y}, C_{y}, D_{y}$ are the elements of the wave matrix in $x$ and $y$ direction respectively.

For each particle, the simulation of the recorded intensity is performed by three basic operations, $i$ ) propagation of the incident wave from the source to the particle plane using Eq. (1) and $A B C D_{x}=A B C D_{1 x}$, $A B C D_{y}=A B C D_{1 y}$ matrices, ii) multiplication of the calculated field, at the z-particle position, by the particle transmission function $\mathrm{T}$, and iii) propagation of the resulting field from the particle plane to the sensor position using Eq. (1) and $A B C D_{x}=A B C D_{2 x}, A B C D_{y}=A B C D_{2 y}$ matrices.

As for free space, the main difficulty is to evaluate numerically the Collins formula. In literature several approaches are presented to address this issue. Among them, Ozaktas expresses Eq. (1) as a magnified FrFT with a phase curvature [5]. Another approach is to derive an analytical expression of the resulting amplitude after the two propagations. Indeed, as demonstrated, by Verrier et al., the amplitude $A_{z}$ can be calculated thanks to an analytical solution by considering an incident Gaussian beam profile [8].

Finally, as for free space, the Collins integral can be rewritten as a convolution product as follow:

$$
A_{z}(x, y)=\frac{\exp (i k z)}{i \lambda A_{x} A_{y} \sqrt{B_{x} B_{y}}} \exp \left(i \pi \frac{C_{x} x^{2}}{\lambda A_{x}}\right) \exp \left(i \pi \frac{C_{y} y^{2}}{\lambda A_{y}}\right)\left[A\left(\frac{u}{A_{x}}, \frac{v}{A_{y}}\right) * h(u, v)\right](x, y)
$$

with $h(u, v)=\exp \left(i \pi u^{2} / \lambda A_{x} B_{x}\right) \exp \left(i \pi v^{2} / \lambda A_{y} B_{y}\right)$ the Generalized Impulse Response (GIR). A Generalized Fresnel Transfer (GFT) function $H$ can be expressed by calculating the Fourier transform of the GIR:

$$
H(u, v)=\exp \left(-i \pi A_{x} B_{x} \vartheta_{x}^{2}\right) \exp \left(-i \pi A_{y} B_{y} \vartheta_{y}^{2}\right)
$$

with $\vartheta_{x}=x /\left(\lambda A_{x} B_{x}\right), \vartheta_{y}=y /\left(\lambda A_{y} B_{y}\right)$ the spatial frequencies along $x$ and $y$ respectively.

Then the evaluation of Eq. 1 is achieved through two successive Fourier transforms:

$$
A * h=F T^{-1}(F T(A) \cdot H)
$$

Required sampling conditions on the supplementary quadratic phase $\exp \left(i \pi C_{x} x^{2} / \lambda A_{x}\right) \exp \left(i \pi C_{y} y^{2} / \lambda A_{y}\right)$ regarding Shannon theorem, bring sampling issues for the propagation (iii) that can be addressed by considering a general system merging the two initial ABCD systems. This generalized convolution method allows to describe the particle transmission function $\mathrm{T}$ without limitations regarding shape and relative refractive index of the particles. A comparison between Verrier approach, GFT approach and experimental acquisition is presented in Figure 1 for the astigmatic experimental setup described in [1]. A good agreement is observed between all holograms.
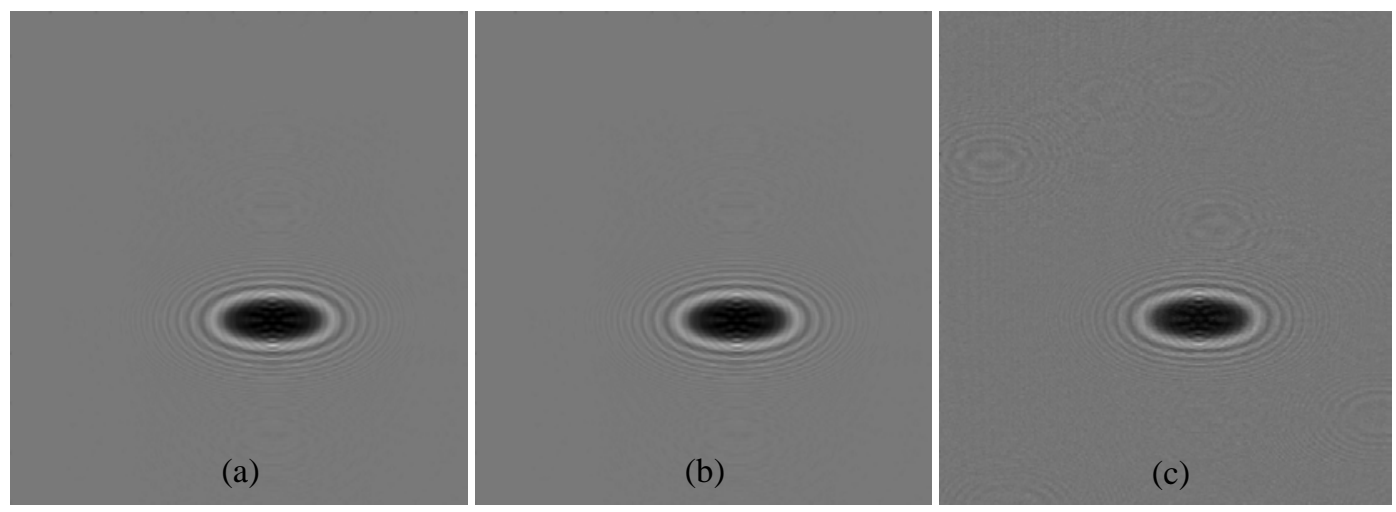

Figure 1 : Intensity recorded for a 1mm diameter oil droplet rising in water through an astigmatic experimental [1] - (a) Analytical solution, (b) GFT, (c) experimental acquisition. 


\section{Particles parameters retrieval}

The particles characterization from a recorded hologram is realized through two main steps.

In the first step, a Best Fitting Approach (BFA) is applied to retrieve the (x,y) position and the diameter $D$ of the particles. This latter consists in a simple least square minimization between the experimental acquisition and the numerical holograms simulated with the approach detailed in the previous section.

The second step consists in finding the best focusing plane along the $z$-axis. To achieve this, the field is reconstructed in successive planes along the optical axis by convoluting the recorded hologram with GIR or GFT. Then, using the photonic jet generated by a particle when interacting with a coherent light, a maximum intensity position $z_{\max }$ is detected thanks to the Tenengrad variance [9]. This maximum corresponds to the "focal length" of an equivalent ball shaped lens. Following these assumptions, the z-position of the center of the particle can be easily expressed as:

$$
z \square z_{\max }-\frac{n D}{4(n-1)}
$$

With $n$ the relative refractive index of the particle.

An example of the reconstruction of a polydisperse population from an experimental hologram acquired in an astigmatic experimental setup is given in Figure 2, in the case of rising oil droplets

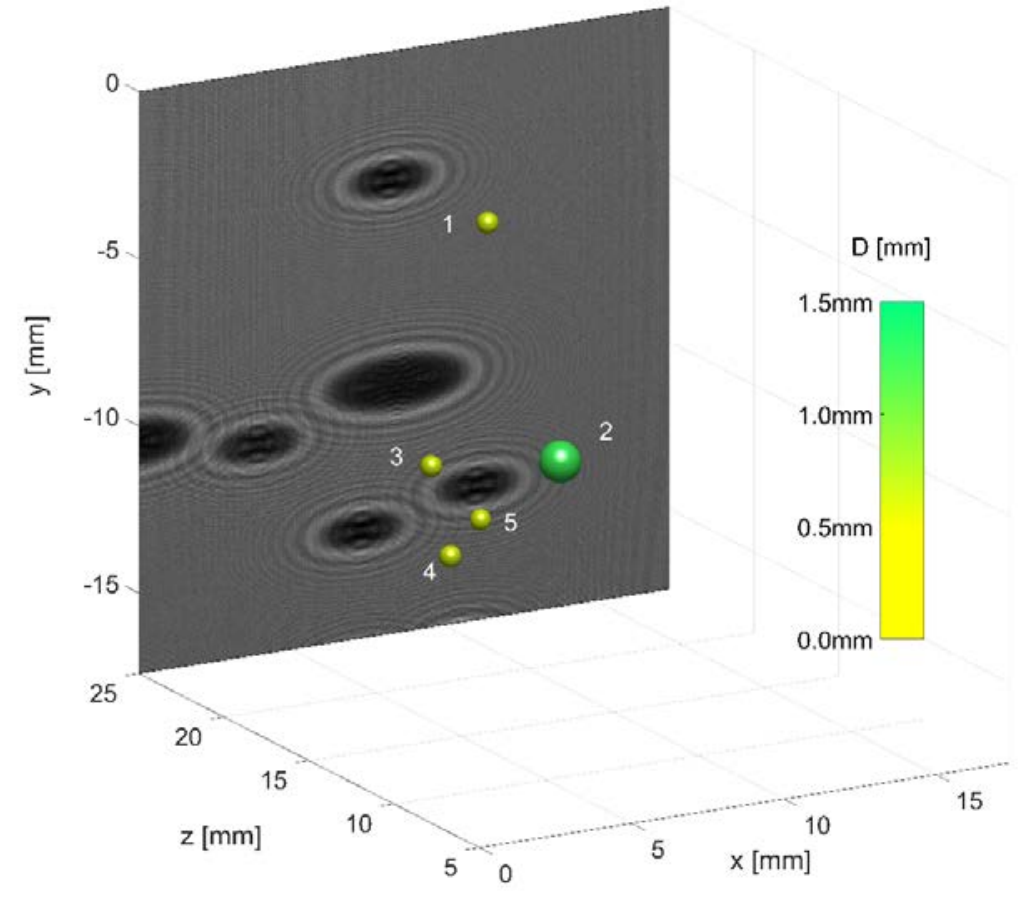

Figure 2: Reconstruction of five rising oil droplets in an astigmatic experimental setup.

\section{References}

[1] Lamadie F, Bruel L, Himbert M. Digital holographic measurement of liquid-liquid two-phase flows. Optics and Lasers in Engineering. 2012;50:1716-25.

[2] Collins J, Stuart A. Lens-system diffraction integral written in terms of matrix optics. JOSA. 1970;60:1168-77.

[3] Verrier N, Fournier C, Méès L, Fournel T. In-line particle holography with an astigmatic beam: setup self-calibration using an "inverse problems” approach. Applied optics. 2014;53:G147-G56.

[4] Palma C, Bagini V. Extension of the Fresnel transform to ABCD systems. JOSA A. 1997;14:1774-9.

[5] Ozaktas HM, Zalevsky Z, Kutay MA. The fractional Fourier transform: Wiley, Chichester; 2001.

[6] Voelz DG. Computational fourier optics: a MATLAB tutorial: Spie Press Bellingham; 2011.

[7] Saleh BE, Teich MC, Saleh BE. Fundamentals of photonics: Wiley New York; 1991.

[8] Verrier N. Microscopie holographique numérique: modélisation et développement de méthodes pour l'étude d'écoulements canalisés et microcanalisés: Université de Rouen; 2009.

[9] Bueno-Ibarra MA, Acho L. Fast autofocus algorithm for automated microscopes. Optical Engineering. 2005;44:063601--8. 